\title{
Recent patents in organoids and organs-on-chips
}

Patent number

US $9,914,920$

A culture apparatus, culture systems and an alternating ionic magnetic resonance electromagnetic chamber for culturing cells, tissues or organoid bodies or for delivering a pulsating alternating ionic magnetic resonance field to an object of interest, including cells, tissues or organoid bodies or to an animal, human or plant. The culture apparatus comprises a culture unit having growth and nutrient modules and a randomizing adapter to continually randomize the gravity vector in the growth module. The culture systems further comprise the alternating ionic magnetic resonance electromagnetic chamber.

US 9,874,285 A platform for cultivation, maintenance, and/or analysis of one or more bio-objects, including one or more integrated bio-object microfluidics modules. Each integrated bio-object microfluidics module is configured to cultivate, maintain, analyze and/or mimic functionalities of a respective bio-object, and includes one or more on-chip pumps; a plurality of fluidic switches; and a microfluidic chip in fluid communication with the one or more on-chip pumps and the plurality of fluidic switches, having at least one chamber for accommodating the bio-object and a plurality of fluidic paths connecting at least one chamber, the one or more on-chip pumps and the plurality of fluidic switches, and a power and control unit adapted for selectively and individually controlling the one or more on-chip pumps and the plurality of fluidic switches for performing bio-object microfluidics functions.

US 9,856,458 A 3D in vitro bi-phasic cartilage-bone organoid, including a layer of an artificial cartilage tissue, and a layer of an artificial bone tissue comprising a structure-giving scaffold and a bone marrow structure. The layer of the artificial cartilage tissue contacts at least one surface of the layer of the artificial bone tissue.

US 9,791,433 A multi-organ-chip device comprising a base layer; an organ layer arranged on the base layer; an antral layer arranged on the organ layer; and an actuator layer; wherein the base layer is configured to provide a solid support for the further layers; the organ layer is configured to comprise a multiplicity of individual organ equivalents, each organ equivalent comprising one or more organ growth sections, each of the organ growth sections being configured to comprise an organoid cavity for housing at least one organoid of an organ and to comprise a micro-inlet and a micro-outlet for fluid communication between the organoid cavity of the organ growth section and a selfcontained circulation system, wherein the organ layer comprises at least one organ equivalent configured to represent the organs lung, small intestine, spleen, pancreas, liver, kidney and bone marrow, respectively, and a self-contained circulation system configured to be in direct fluid communication with the organ growth sections of the organ layer via the micro inlets and outlets of the organ growth sections; the antral layer is configured to comprise a multiplicity of cavities and tubes arranged to be in fluid communication with selected organ equivalents or organ growth sections in order to allow for exchange of fluids between cavities and organ growth sections; and the actuator layer is configured to comprise a multiplicity of actuators arranged and configured to regulate a pressure force applied on a selected organ equivalent, the self-contained circulation system and/or part thereof.

US 9,725,687 Integrated organ-on-chip microphysiological systems, representations of living organs and support structures for such microphysiological systems.
US 9,696,304 Methods for detecting pathogens, infectious diseases, and physiological conditions by quantifying change of impedance over time of when a biological sample is applied onto a lab-on-a-chip. The lab-on-a-chip utilizes alternating-current electrokinetic phenomena such that molecules move or are carried in an electric field generated by the application of an electrical signal of predetermined magnitude and frequency to an electrode array of the lab-on-a-chip.

US 9,680,970 A method for a bridge device to interface between an external device and an implantable medical device (IMD). The bridge device includes a system on a chip (SoC) having a memory, an input/output interface, a standard wireless computer network (SWCN) controller and a bridge controller integrated into a single integrated circuit. The method includes configuring the bridge controller to convert data between a medical implant communication service (MICS) protocol and a SWCN protocol, coupling a MICS controller to the SoC, and configuring the MICS controller to manage operation of a first transceiver based on the MICS protocol. The method includes configuring the SWCN controller to manage operation of a second transceiver based on the SWCN protocol, communicating between the bridge device and an IMD utilizing the first transceiver, and communicating between the bridge device and an external device utilizing the second transceiver.

\section{Assignee}

Inventor

Date

Goodwin TJ,

Goodwin TJ,

Kushman MJ

Kushman MJ

$3 / 13 / 2018$

Vanderbilt

University

(Nashville, TN,

USA)

Block III FE,

Samson PC, Werner EM,

Markov DA, Reiserer RS,

Mckenzie JR, Cliffel DE, Matloff WJ, Block Jr. FE, Scherrer JR, Tidwell WH, Wikswo JP

TissUse (Berlin) Rosowski M, Kadler S, Lauster R, Marx U

President and Fellows of Harvard College (Cambridge,

MA, USA), Vanderbilt University (Nashville, TN, USA)

University of

Tennessee

Research

Foundation

(Knoxville, TN, USA)

Pacesetter, Inc. Wu Y, Yang J, (Sunnyvale, CA, Shankar B, Young C-W, USA)
Wikswo JP, Samson PC, Block III FE,

Reiserer RS, Parker KK, McLean JA, McCawley LJ, Markov D, Levner D, Ingber DE, Hamilton GA, Goss JA, Cunningham R, Cliffel DE, McKenzie JR, Bahinski A, Hinojosa CD Wu J, Eda S Shahandeh R 